\title{
Vortex valve
}

\author{
Karel Adámek ${ }^{1, *}$, Jan Koláŕr ${ }^{1}$, and Pavel Peukert ${ }^{1}$ \\ ${ }^{1}$ VÚTS Liberec, a.s., Department of Flow Simulations, Svárovská 619, 46001 Liberec, Czech Republic
}

\begin{abstract}
The paper deals with a flow field inside the so-called vortex valve, used as an outlet device on retention reservoirs for retention of rainstorms and later slow outflow into sewerage etc. The system is very simple, without moving parts. Using the method of numerical flow simulation, the unusual flow characteristic $\Delta \mathrm{p}=\mathrm{f}(\mathrm{Q})$, containing two branches, is explained. Further, there it is studied influence of both inlet/outlet opening sizes on the form of the characteristic. Results can be used for designing the new model series of valves for various flows.
\end{abstract}

Keywords: Flow numerical simulation, Vortex valve, Flow characteristic.

\section{Introduction}

The aim of the presented study is the statement of the resistance-flow characteristic $\Delta \mathrm{p}[\mathrm{Pa}]=\mathrm{f}\left(\mathrm{Q}\left[\mathrm{m}^{3} / \mathrm{s}\right]\right)$ for the so-called vortex valve, mounted usually on the outlet from the retention reservoir. Such a reservoir is used for instance as the retention of the rainstorm or similarly and for the consecutive slow outflow into drainage etc. The influence of main design parameters, first of all inlet/outlet diameters, is discussed, too, as guidelines for practical commercial design.

From a simple search, some essential information, only, is going out. The principle is described in [1], but the presented geometry is very complicated. Next studies [2], [3] describe theory and contain next 50 references, approx. No more references are added, because the presented results were made by own simulations and measuring and using standard knowledge of fluid mechanics, well known for technical community. It is a pity that this knowledge is not used in daily practice.

Some actual designs are presented in many internet references, but as commercial messages, only and the principle is described curiously by Mr. Google and many dealers and producers, for instance as "the effect is given by flow phenomenon" or "it works on the principle of hydraulic phenomenon". Reality is not so mysterious, the flow inside of such a valve is possible to describe by numerical flow simulation, completed by some essential formulas of fluid mechanics.

\section{Initial model}

\subsection{Geometry}

The model contains six liquid bodies, see Fig. 2-1. The surrounding retention reservoir is not modelled here - it is assumed that the velocity range in such surrounding can be neglected in comparison with the flow inside the studied valve.
1. Inlet - cylindrical tangential, situated from below.

2. Chamber - cylindrical, with tangential inlet and axial outlet.

3. Outlet - axial hollow on the forefront.

4. Outlet flange - outlet prolongation.

5. Vent opening - small orifice in the highest point of the chamber.

6. Outer surroundings (air) - is minimalized here, a smaller volume of the model means shorter time of the solution. In this model, the flow resistance of the valve is solved, only, the shape of the outer flow (or its spraying quality) is not important here.

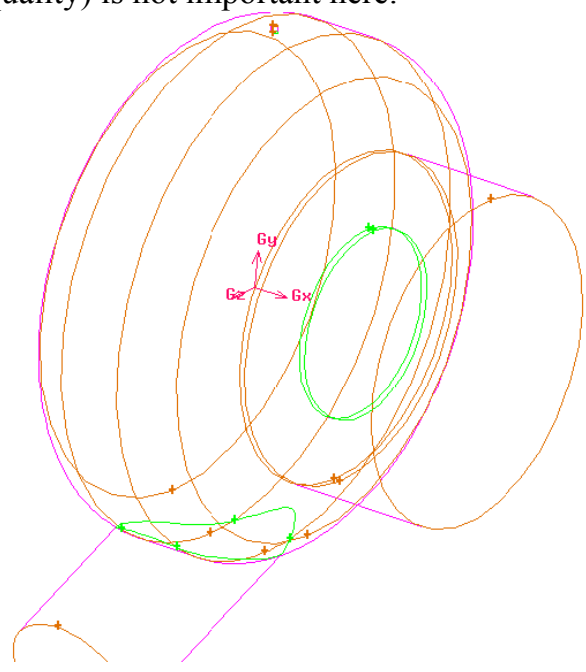

Fig. 2-1. Model composed from six liquid bodies.

\subsection{Mesh}

Regarding the complicated two-phase flow it is necessary to use a fine calculating mesh after Fig. 2-2, first of all, in the vicinity of the outlet, where the flow is disintegrating. The initial mesh can be modified yet during calculation.

\footnotetext{
Corresponding author: k.adamek@,volny.cz
} 


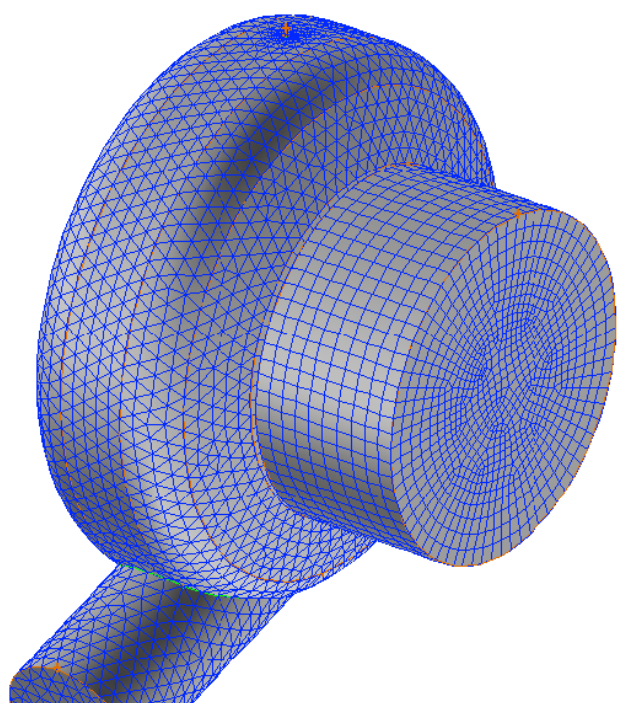

Fig. 2-2. Mesh of the model.

\subsection{Boundary and initial conditions}

In the inlet cross-section, there it is simply defined constant (hydrostatic) pressure, given by the height of the surrounding water level. Zero pressure is defined here in the middle of this inlet cross-section. For the used dimensions of the valve, the water level on the outlet axis reaches of $0.2 \mathrm{~m}$ approx. (pressure $2 \mathrm{kPa}$ ) and at the upper contour of the chamber $0.36 \mathrm{~m}$ of water approx. (pressure $3.6 \mathrm{kPa}$ ).

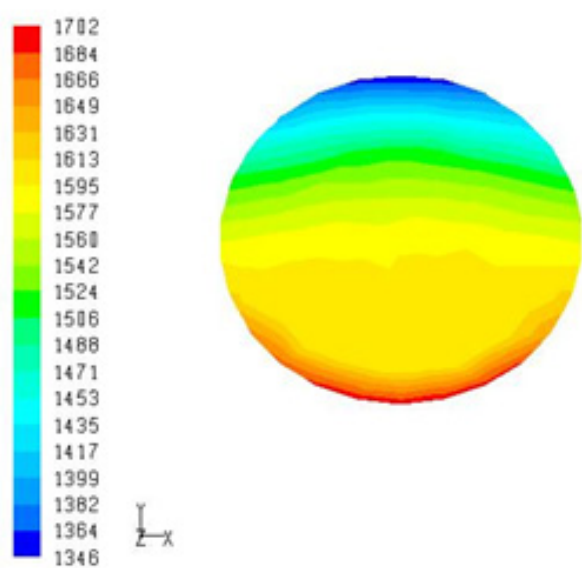

Fig. 2-3. Uneven inlet pressure field (water level of $0.16 \mathrm{~m}$ ).

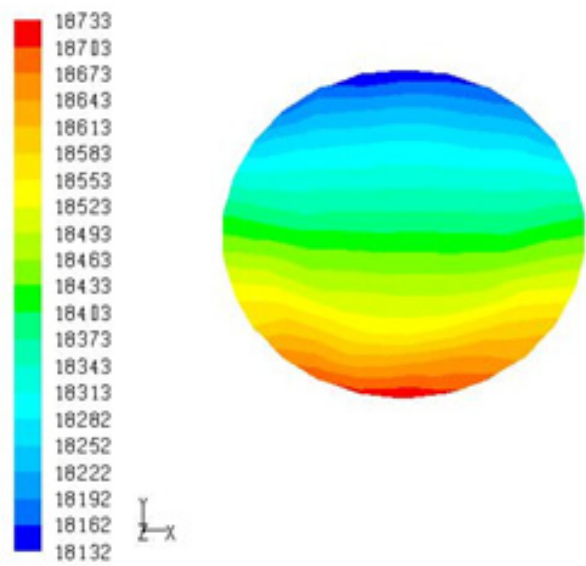

Fig. 2-4. Uneven inlet pressure field (water level of $2.0 \mathrm{~m}$ ).
Really, the pressure distribution is not absolutely constant here, this influence is neglected hereafter. Fig. 2-3 and Fig. 2-4 present real pressure fields in the inlet cross-section for min. and max. water levels of $0.16 \mathrm{~m}$ and $2.0 \mathrm{~m}$ - some part of the defined static pressure is consumed yet for kinetic energy of the flow.

In the surroundings, there is zero pressure (atmosphere).

Operational condition - gravity acceleration of $9.81 \mathrm{~m} / \mathrm{s}^{2}$ in the down direction - axis (-y).

Initial conditions: the mixture pressure in the inlet max. $20 \mathrm{kPa}$ and the value of the phase $\mathrm{f} 2$ is equal to zero (water only), in the outlet, the mixture pressure 0 $\mathrm{Pa}$, with defined backflow of the phase 2 equal to 1 (air only).

\subsection{Solver}

Isothermal model (if speculating about friction losses, the water warming would be of some $0.01 \mathrm{~K}$, only). Turbulence model k- $\omega$ SST.

Two models of two-phase (water-air) flow were tested: the unsteady model „VOF“ (volume of fluid) with a very short time step and the steady model „mixture“.

\subsubsection{Model VOF (volume of fluid)}

As an illustration, only, the testing images of both phases after several first time steps of the solution are presented in Fig. 2-5 (low pressure difference) and in Fig. 2-6 (high pressure difference). The solution with a time step of $10 \mathrm{~ms}$ is diverging so that the time step shortening to $0.1 \mathrm{~ms}$ approx. should be necessary.

The field of both phases contains more details, captured in the actual time step of the unsteady solution. Using small pressure (Fig. 2-5), the water (blue, phase f2 $=0$ ) is flowing mainly down, owing to gravitation inside, there is not any important rotating flow yet. In the surrounding, there is air (red, phase $\mathrm{f} 2=1$ ). Using a higher pressure (Fig. 2-6), the axial flow becomes more dominant.

The large surroundings volume (right) is deleted later, due to a shortening of the solution time.
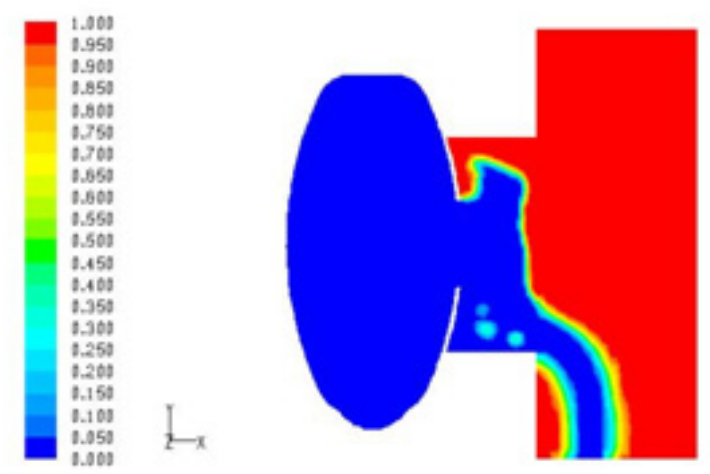

Fig. 2-5. Phase interface, low pressure difference, $0.7 \mathrm{~s}$ after start (model COUPLED). 


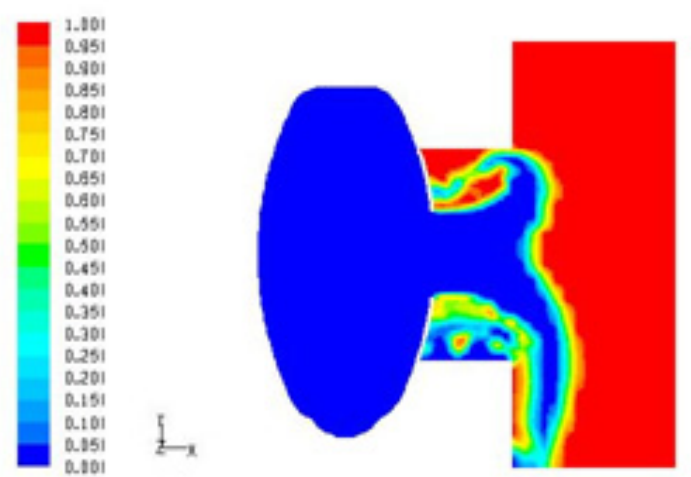

Fig. 2-6. Phase interface, high pressure difference, $0.35 \mathrm{~s}$ after start (model SIMPLE).

\subsubsection{Model "mixture"}

The results of the first simulation are not presented here; they were used for verification of the proper progress of the solution and are used in the models below. From the results, it follows that the interface water-air is changing in the time so that the presented result is any fictive steady flow field, captured in any actual time of the solution. The relatively large boundary water-air is given by this unsteady time-dependent water level (surface).

\subsection{Conclusions for the next models}

Pressure difference (instead of water level height) is defined in the middle of the inlet cross-section. Difference between lowest and highest point of this cross-section can be neglected toward other dimensions.

The steady two-phase model "mixture“", is used in preference. Above all, because the calculation of each point of the operational characteristics is converging faster and the balance error (difference between inflow and outflow) is small enough. The flow value of the converged solution is oscillating a little; therefore, the recorded values can be time-different in the range of this oscillation.

Two-phases model VOF - if the method is used from the initial state, it is necessary a very short time step of the solution, $0.1 \mathrm{~ms}$ and less, and the time of the solution is very long. So it is better to use this method as a finishing of the previous steady model ,mixture“. Then, the time step of $0.01 \mathrm{~s}$ can be used and the solution finishing is real. The values of the flow, received with the time step of $10 \mathrm{~ms}$, are of $6-7 \%$ smaller in comparison with the method ,mixture“ and with the time step of $0.1 \mathrm{~ms}$ of $3 \%$ smaller. Probably, it is some numerical mistake between both used models - without next investigation.

Vent hole - the solution is unstable when water reaches the level of this hole. Really, it makes no problem, but during the simulation, only. Maybe, using a larger diameter of the orifice, the simulation could be more stable.
The primary aim is the creation of the resistance characteristics of the valve $\Delta p=f(Q)$, the shape of the water splashing in the surroundings is not important. A prospective error in consequence of an unsuitable twophase model will be the same for all solved cases; therefore, the mutual comparison of all cases remains in the same rate.

As a piece of information, only, the converged solution by the method „mixture“ needs many time steps, using the method VOF. An ideal procedure is the beginning with „mixture“ and to continue with VOF. Then, for higher water levels, the time step of $10 \mathrm{~ms}$ is sufficient, but when the chamber is not full, it is necessary to shorten the time step at least to $0.1 \mathrm{~ms}$. Thereby, the solution time is considerably longer.

The definition of the 2-branches characteristic of the observed valve needs for each geometry to simulate as minimum $4+4$ cases for various pressures.

Flow resistance of outlet flange: The check simulation of one case confirms that for the model without flange, the flow is of $3 \%$ higher approx. It means that the main resistance of the valve is creating inside the valve, the flange influence is not important.

For the shortening of the solution time for the next models, the outer area behind the flange (air area) is removed and bigger mesh elements are used. The aim of the solution is to observe water flow inside the valve, not outside.

\section{Basic model}

Following the results of the initial model above, the steady model of two-phases flow ,mixture“ is used, the steady shape of the water level is displayed.

The thick interface water/air represents the real level waviness; therefore, the time-averaged positions of the water level are displayed. The tangential bottom inlet creates rotational flow, but at a partially filled chamber, the water level is very corrugated. This action is unsteady and the used steady model ,mixture“ records any steady state in any actual time.

\subsection{Flow fields for various water levels}

For various water levels, there are presented the interfaces water/air (blue/red). For the low water levels, the water is flowing from the bottom tangential inlet (crosswise to the paper plane) directly into the axial outlet - a typical vessel outflow, for higher water levels, the rotating flow in the chamber is creating. All Figures are presented as lengthwise vertical cross-sections through the outlet axis. 


1.111
1.951
1.951
1.851
1.841
1.751
1.711
1.851
1.641
1.551
1.541
1.451
1.411
1.351
1.311
1.251
1.211
1.151
1.111
1.151
1.111

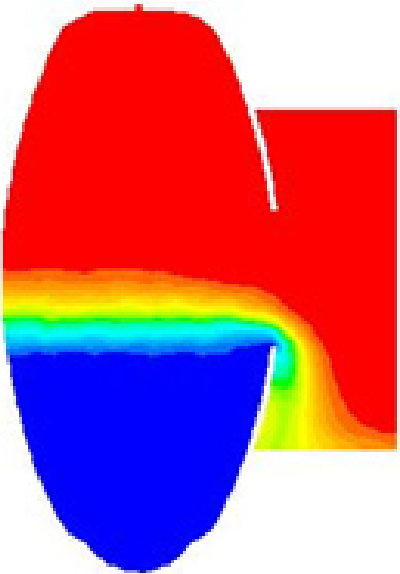

Fig. 3-1. At the lowest possible water level, the water hardly flows over the bottom edge of the outlet directly down on the bottom surface of the flange; the surrounding air is coming in from the right surroundings.
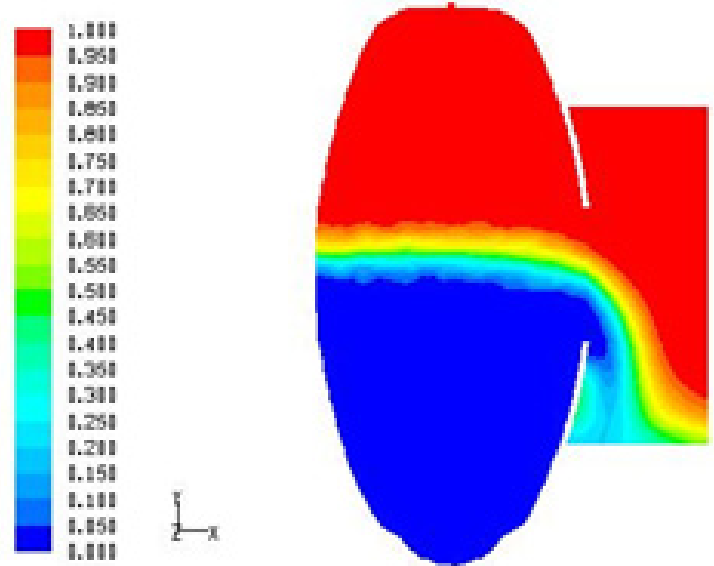

Fig. 3-2. Water level is defined just on the outlet axis, situation is similar, and the outflow is more intensive. The level (theoretically horizontal) is inclined to the outlet.
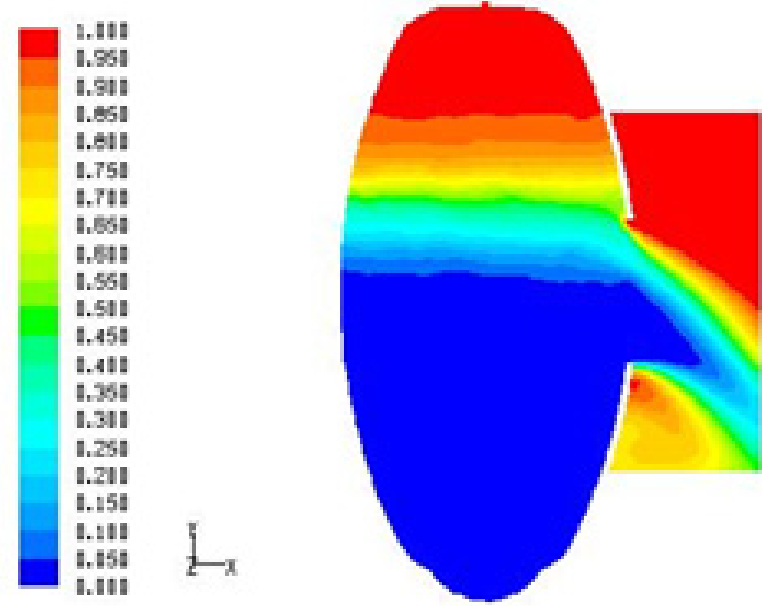

Fig. 3-3. Water level defined at the upper edge of the outlet, the flow is separated from the chamber wall, it is visible the beginning rotating flow, the air flow in the vent hole practically does not flow out.

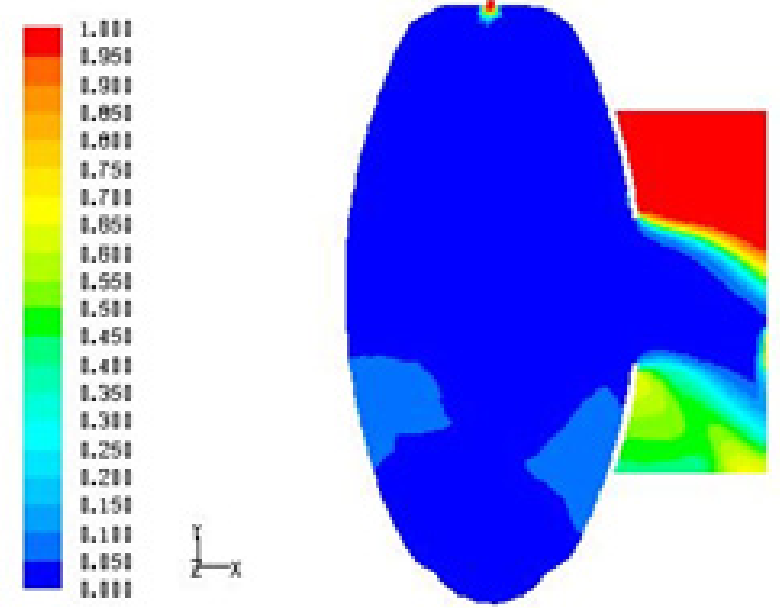

Fig. 3-4. Water level just under the upper edge of the chamber, the rotating flow inside is transformed into axial outer flow; the gravity influence is visible yet.

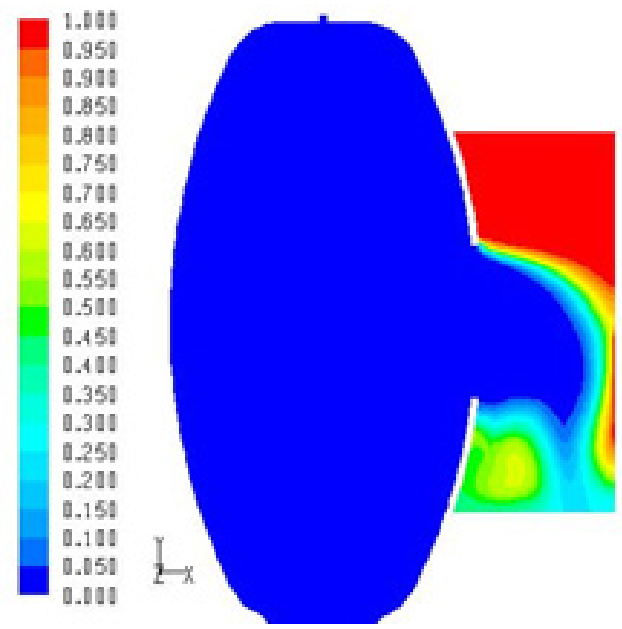

Fig. 3-5. Water level just over the upper edge of the chamber, the expressive rotating flow inside; outside it begins the transformation of gravitational flow into water splashing (spraying).
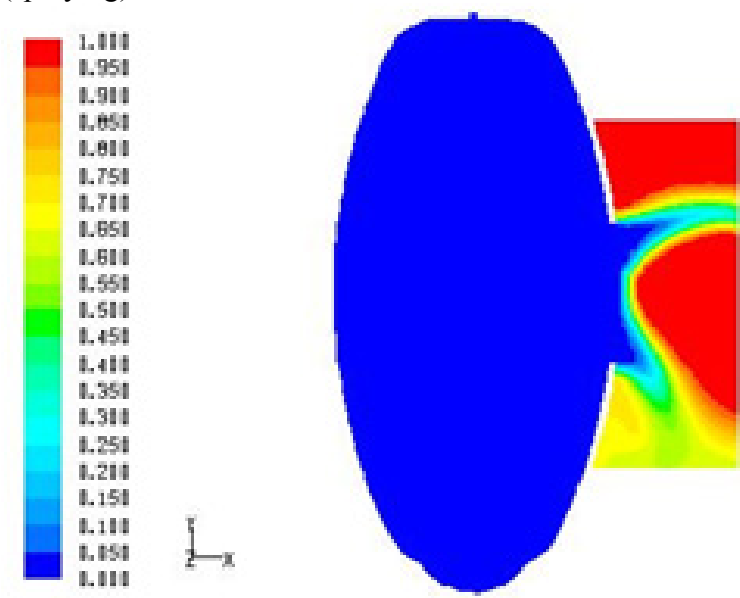

Fig. 3-6. The expressive inner rotating flow causes the spraying of the axial water flow into a conical shape, in the middle filled by back flowed air, deformation due to the gravity. For the chamber of a larger outlet diameter (see below), the air is coming up to the chamber interior. 


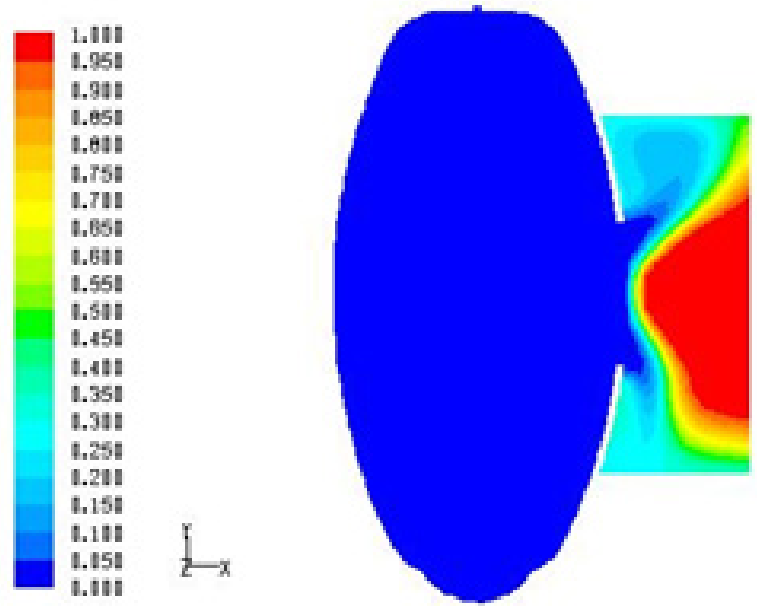

Fig. 3-7. At a higher water level, the expressive rotating flow is persisting, the conical water spray partially hits on the inner wall of the flange and is coming back. The gravity influence is less, but still evident it is the some flow along the bottom wall of the flange.
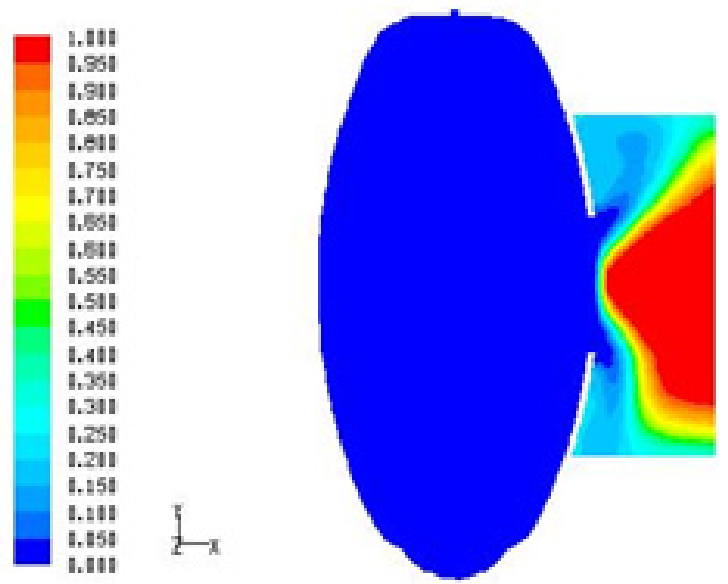

Fig. 3-8. Continuation of the previous for the max. defined water level (pressure). The higher part of the sprayed flow hits the inner walls of the flange and is rotating there.

\subsection{Resistance characteristic}

For the several water levels (pressure differences), relevant cases were solved, see above Fig. 3-1 to Fig. 38 . From the received results, the flow characteristic

$$
Q\left[\mathrm{~m}^{3} / \mathrm{s}\right]=f(\Delta p[\mathrm{~Pa}])
$$

was evaluated and the inverse resistance characteristic respectively

$$
\Delta p[P a]=f(m[\mathrm{~kg} / \mathrm{s}]) \text { resp. } \Delta p[P a]=f\left(Q\left[\mathrm{~m}^{3} / \mathrm{s}\right]\right),
$$
see the graph in Fig. 3-9.

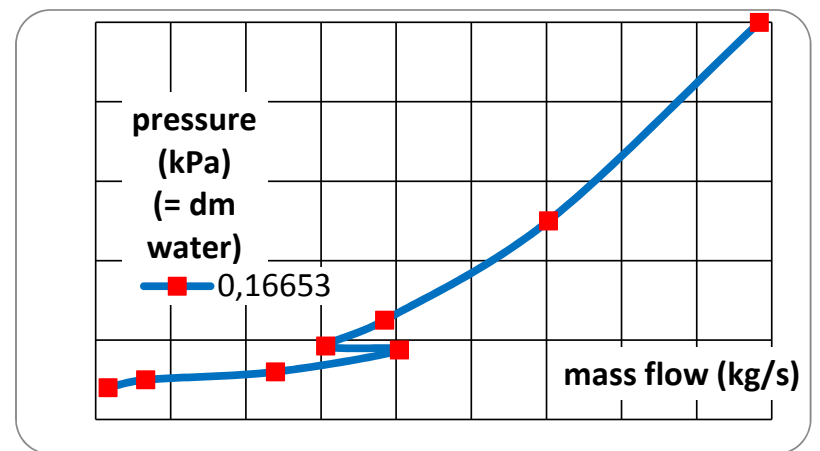

Fig. 3-9. Resistance characteristic of the vortex valve (numerical simulations).
The characteristic has two parabolic branches of different gradient. The „switching-over“ is coming when the water level is just around the upper outline of the chamber. Flow through the system without rotation (or with partial rotation, only) is passing into the flow of fully created rotating flow. The numerical solution in this ,Switching-over“ area is not stable, the stabilization needs a longer calculating time. Probably, the instability is given by an alternating air flow in and out through the small vent hole, which is given by a wavy water level. Such a small orifice acts as a large flow resistance and the response on the chamber filling is delayed. Maybe, more stable solution could be possible with a large vent diameter (not tested). Another influence is the transition into the fully rotating flow.

For a better description of this area it should be created more calculations. For the smallest flows, the curve gradient is higher - at the lowest water level the flow width is much less than the outlet diameter.

There are visible two different modes of the flow:

Flow with free water level inside the chamber (smaller water level, smaller gradient of characteristic) neglecting the flow resistance in the chamber, the theoretical flow velocity $\mathrm{w}[\mathrm{m} / \mathrm{s}]$ and volume flow $\mathrm{V}$ $\left[\mathrm{m}^{3} / \mathrm{s}\right]$ at water level $\mathrm{H}[\mathrm{m}]$ can be solved from simple formulas

$$
\begin{gathered}
w=\sqrt{ }(2 . g . H) \\
V=\pi / 4 . D^{2} . w .
\end{gathered}
$$

Flow without free water level inside the chamber (higher water level, higher characteristic gradient) - water from the tangential inlet is rotating inside the chamber and on the outlet edge, the compact flow is sprayed into individual drops.

\subsection{Detailed analysis of the results}

For an absolutely free flow, the theoretical outlet velocity is logically higher, comparing with the real flow velocity in a real valve with flow resistances. The comparison of such a theoretical value with a simulated value is in Fig. 3-10.

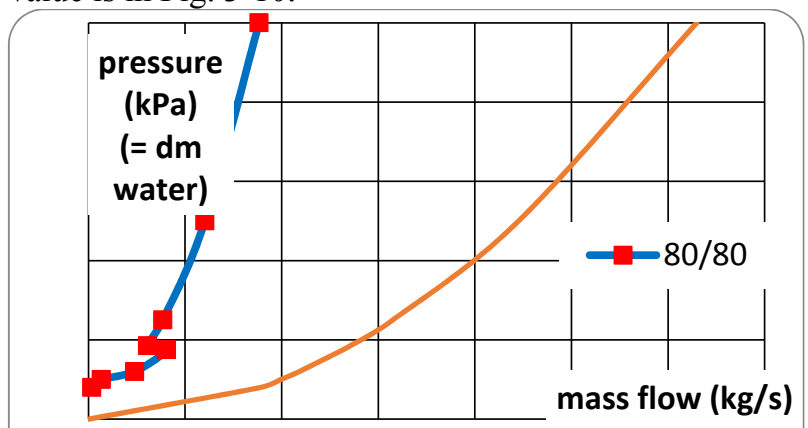

Fig. 3-10. Real (low) mass flow through the simulated valve and ideal (high) mass flow through the simple opening.

Further, there is derived the so-called flow coefficient

$$
\varphi=w_{\text {real }} / w,
$$

containing all losses of the valve (inlet, friction in the inlet tube, inlet in the chamber, rotation and friction in 
the chamber, passing into the outlet, flow in the outlet, flow separation, all as three-dimensional flow). The received values of flow coefficient for the observed case are presented in Fig. 3-11.

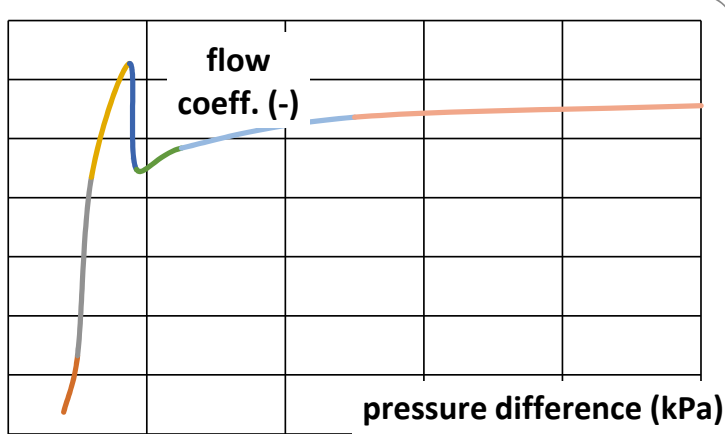

Fig. 3-11. Flow coefficient for the case from Fig. 3-10.

For high flows, the value is approaching to 0.28 and with decreased flow the value is decreased to the value of 0.2. For small flows, the maximum value is 0.31 and with flow decreasing, the value is decreasing quickly, first of all, for a water level under $0.2 \mathrm{~m}$, where the cross-section of the outlet is decreasing in the transverse direction, too. Extrapolating the simulations up to a very high value of $200 \mathrm{kPa}$, we can state that the flow coefficient does not exceed the value of 0.35 approx.

\subsection{Remark to the effect of the vortex valve}

Fig. 3-12 presents the profile of dynamic pressure in the vertical cross section through the outlet plane for the previous case. In the large central part, the pressure is equal to zero (atmospheric). At the periphery, only, the values of $1.2 \mathrm{kPa}$ (negative $\mathrm{x}$-axis, down, here left) and $0.6 \mathrm{kPa}$ (positive $\mathrm{x}$-axis, up, here right) respectively are reached - due to the gravitation, the flow is slightly bent down. Mass flow of $8.8 \mathrm{~kg} / \mathrm{s}$.

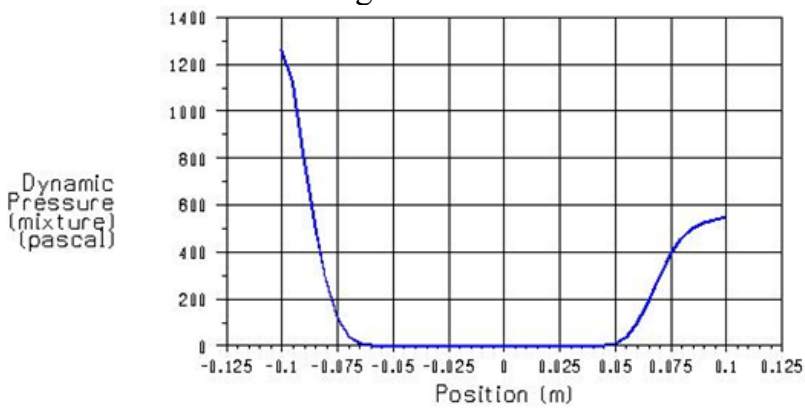

Fig. 3-12: Profile of the dynamic pressure in the vertical plane of the outlet section - a model of the vortex valve

The next Fig. 3-13 presents for the comparison, only, the similar graph of dynamic pressure for a simple cylindrical outlet. In this model, the outlet dimensions and the pressure difference are the same, but the rotational effect is none. The result is typical for sharp outlet flow - the maximum of $18 \mathrm{kPa}$ for mass flow of $23.8 \mathrm{~kg} / \mathrm{s}$, i.e. 2.7 times higher, relative to the previous Fig. 3-12. Decreasing the outlet at $50 \mathrm{~mm}$ approx., the mass flow could be decreased at the above mentioned value of $8.8 \mathrm{~kg} / \mathrm{s}$ and the dynamic pressure could be theoretically $2.5 \mathrm{kPa}$ approx., so still higher, comparing with the vortex valve in Fig. 3-12. The value down (here left) is slightly higher then up (here right) again, due to the gravitation effect.

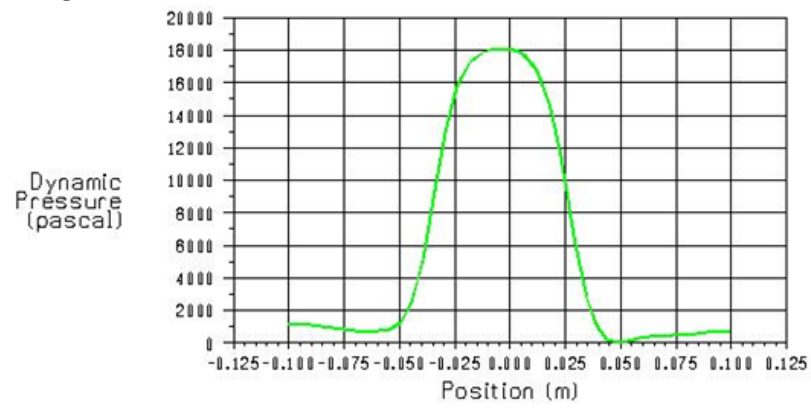

Fig. 3-13: Profile of the dynamic pressure in the vertical plane of the outlet section - a model of the straight outlet

\section{Outlet changes}

After the detailed analysis in Par. 3 above, the next similar solutions were made for valves of other outlets. Using suitable outlet inserts for the same valve body, it is possible to get the range of flow up to $6.25: 1$. Similar sets of flow fields as above are not presented here again, due to their large extent.

Reduced outlet is operating as a classical nozzle, used as the flow acceleration. The characteristic shape is fluent, at higher water levels, the outlet flow remains compact and rotating, with a slight bending due to the gravity, but without spraying, see Fig. 4-1 and Fig. 4-2 for pressure gradients of $2 \mathrm{kPa}$ and $20 \mathrm{kPa}$.
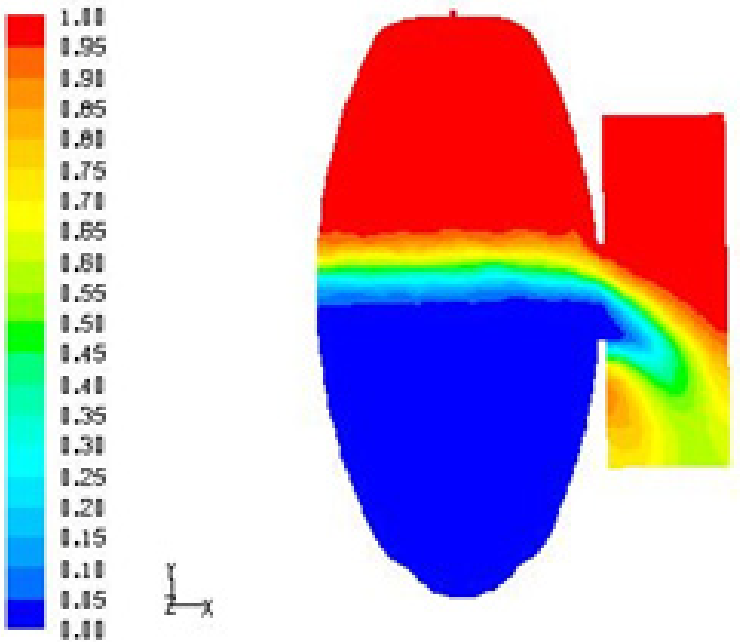

Fig. 4-1. Phase interface, $\Delta \mathrm{p}=2 \mathrm{kPa}$, reduced outlet.

An unsuitable strong dynamic effect of the outgoing flow on the walls in the surroundings. 


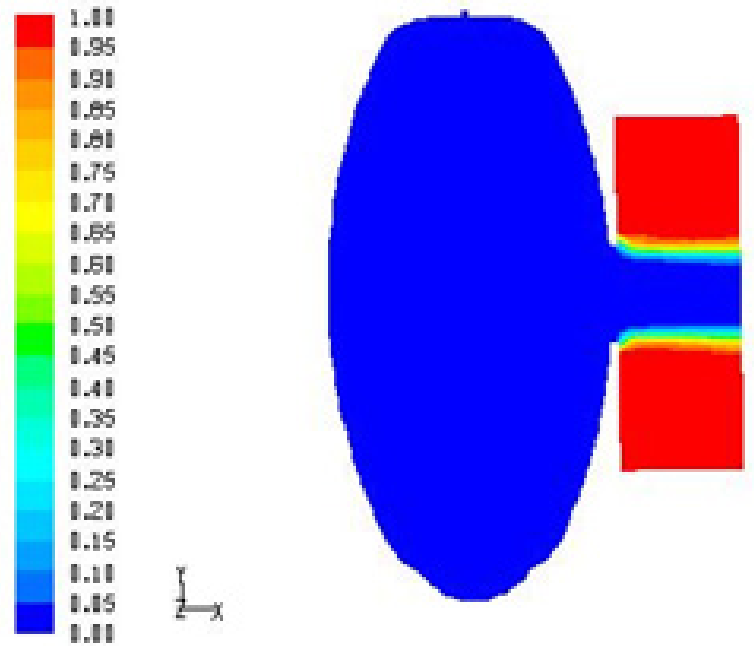

Fig. 4-2. Phase interface, $\Delta \mathrm{p}=20 \mathrm{kPa}$, reduced outlet.

Using the enlarged outlet cross section, inside the inner volume, there it is created an expressive rotating water ring. The outside air is coming inside along the rotation axis, the air amount is greater than that for original outlet diameter. Fig. 4-3 and Fig. 4-4 present the situation for the outlet area $2 \mathrm{x}$ larger in comparison with the basic case from Par. 3 .
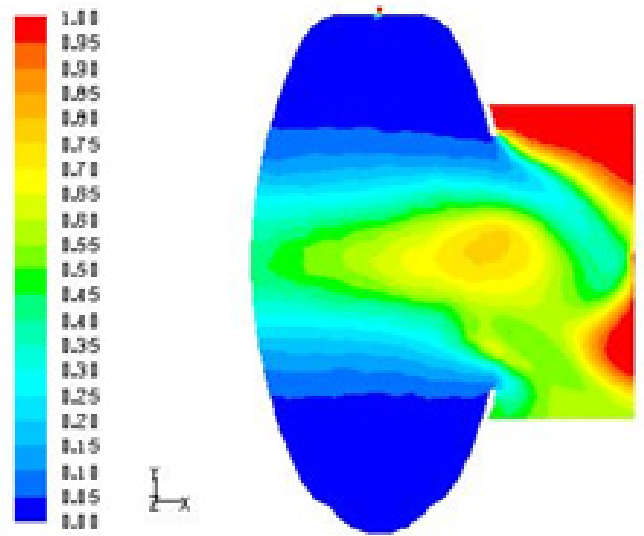

Fig. 4-3. Phase interface, $\Delta p=2 \mathrm{kPa}, 2 \mathrm{x}$ enlarged outlet area.
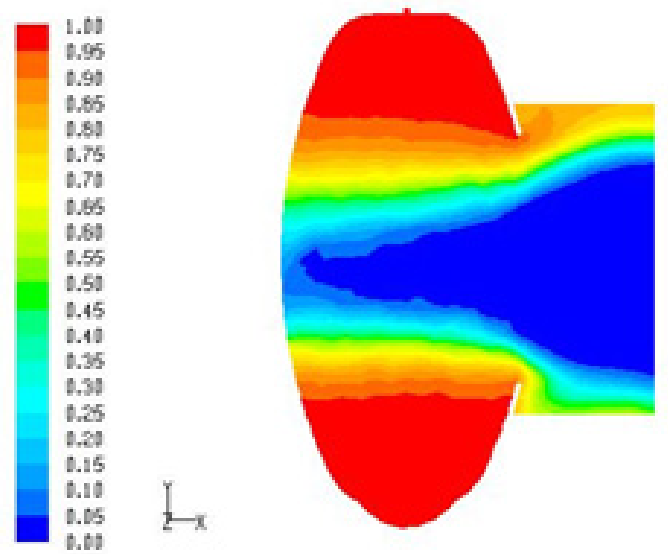

Fig. 4-4. Phase interface, $\Delta \mathrm{p}=20 \mathrm{kPa}, 2 \mathrm{x}$ enlarged outlet area. (Attention, error: here, only, the water is red and the air is blue!)

Using the 4x larger outlet cross section (Fig. 4-5 and Fig. 4-6), the simulation is unstable for pressure under $3.5 \mathrm{kPa}$, i.e. for water level under the upper outline of the chamber. The pressure field is not uniform, too, due to the very large outlet cross-section, the rotating water ring is not being created. Along the axis, there is a visible large area filled by outside air.
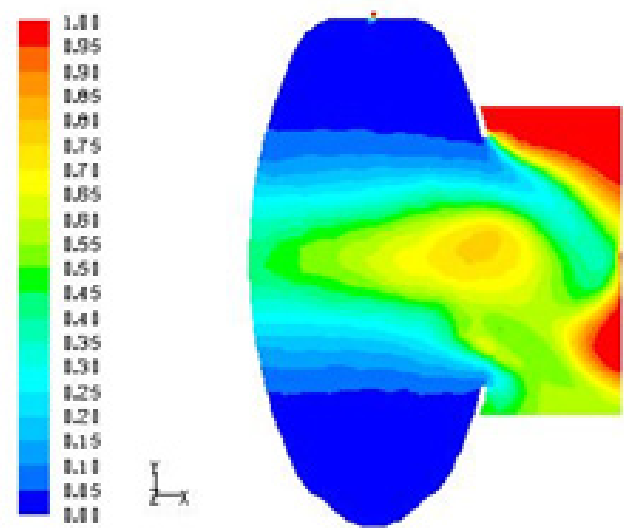

Fig. 4-5. Phase interface, $\Delta \mathrm{p}=3.5 \mathrm{kPa}, 4 \mathrm{x}$ enlarged outlet area.
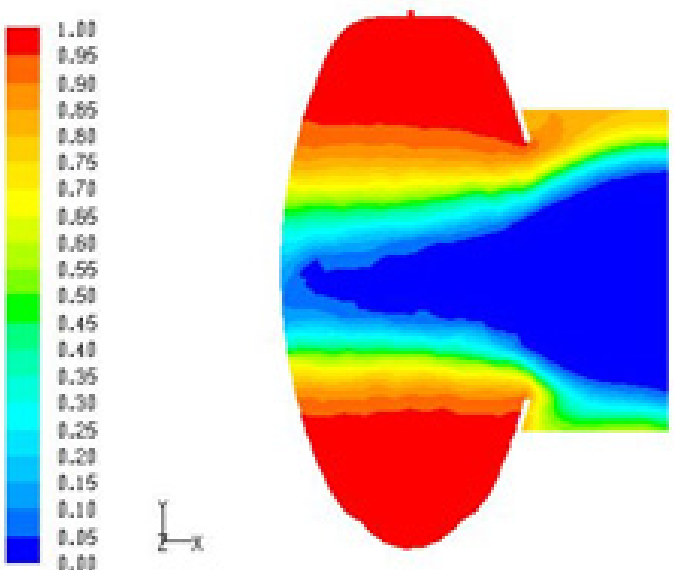

Fig. 4-6. Phase interface, $\Delta p=20 \mathrm{kPa}, 4 \mathrm{x}$ enlarged outlet area. (Attention, error: here, only, the water is red and the air is blue!)

Remark: The usable effect of a dynamic pressure suppressing remains, the spare quality is not important here.

\subsection{Summary characteristics}

The summary of all long-lasting simulations with the resistive characteristics of all solved cases is the sole graph in Fig. 4-7.

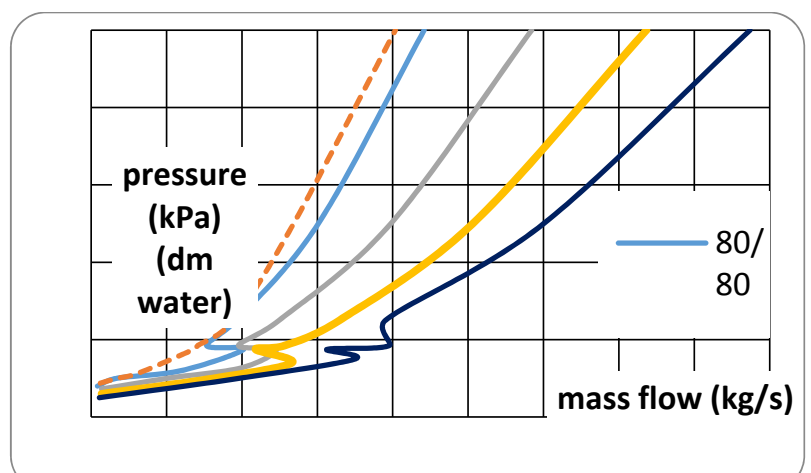

Fig. 4-7. Summary characteristics - changes of the outlet cross-section. 
The geometry remains the same; different are the outlet diameters, only. In the unstable transitional area between two branches of each characteristic, the numerical errors are higher than in the rest of each characteristic, containing two branches.

The Graph in Fig. 4-8, derived from the previous simulations, can be used for a prediction of an outlet diameter for the needed mass flow. In linear correlation it means $\mathrm{y}=$ outlet diameter, $\mathrm{x}=$ needed mass flow.

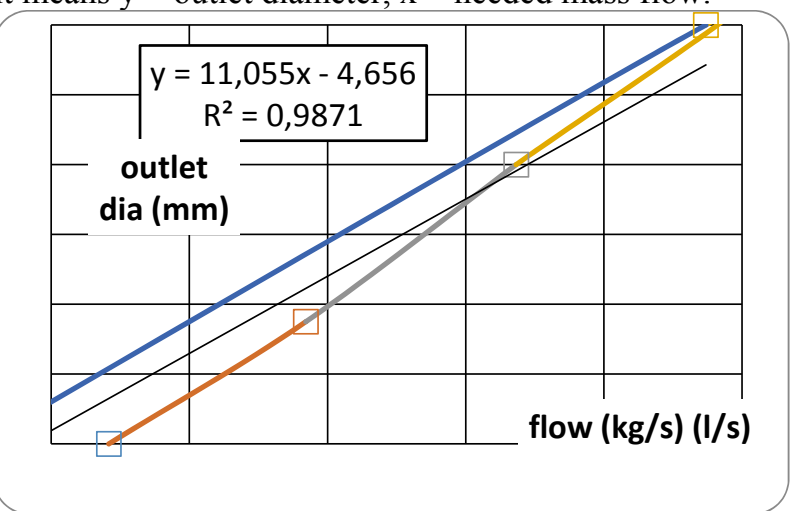

Fig. 4-8. Simple choice of an outlet diameter from the needed mass flow - from the simulations above.

\section{Testing measurement}

The results of the presented numerical flow simulations could be useful for the designing and for a subsequent production of vortex valves. Therefore, the results of the simulations should be verified by real measurements.

The tested valve created after Par. 3 with one inlet cross section and with several outlet cross sections, realized as exchangeable inserts as $1-2-4 \mathrm{x}$ larger inlet cross sections. The water level maintained at several certain values, max. $2.0 \mathrm{~m}$. At the outlet, there it is a mounted measuring device.

For the simulations, there were used water levels of $0.5-1.0-2.0 \mathrm{~m}$, for a measurement with larger outlets, but the used pump allows the level 1.6 or $1.2 \mathrm{~m}$, only, so the results of the simulations are extrapolated for those levels.

The results of the measurements were confronted with the results of the simulations (Fig. 4-7). Comparison of both procedures, simulation and measuring, is in Tab. 5-1.

Table 5-1. Simulations compared with measurements (inlet 80 $\mathrm{mm})$.

\begin{tabular}{|c|c|c|c|c|}
\hline out/in & level & \multicolumn{2}{|c|}{ flow } & diff. \\
\hline- & $\mathrm{m}$ & \multicolumn{2}{|c|}{ l/s } & measur.-simul. \\
\hline & & measur. & simul. & $\%$ \\
\hline 1 & 2.0 & 9.2 & 8.8 & $\mathbf{+ 4 . 5}$ \\
\hline 1 & 1.6 & 7.6 & 8.0 & $\mathbf{- 5 . 0}$ \\
\hline 2 & 1.6 & 10.6 & 10.6 & $\mathbf{0 . 0}$ \\
\hline 4 & 1.2 & 12.0 & 11.55 & $\mathbf{+ 3 . 9}$ \\
\hline
\end{tabular}

The coincidence of both procedures is very good, the error does not exceed the error of standard operating measuring devices $( \pm 4 \%)$. The found differences can be caused by several influences:

- The water level before the valve was not absolutely constant, as well as the water level after the outlet, where the flow meter was installed.

- A next error is given by the definition of zero pressure - constant for simulation, slightly uneven for real measuring, see Fig. 2-3 and Fig. 2-4 above.

- Some error it is brought in the used method of numerical simulation, for instance the coarse mesh elements.

Following such a positive comparison, it is possible to suppose that the application of a numerical flow simulation in the vortex valve is the suitable method for determining the valve main dimensions, operating at the defined flow. Further, it can be supposed that for the geometrically similar shapes of the valves, their operational characteristics are similar, too.

\section{Conclusion}

For a determination of resistance characteristic $\Delta \mathrm{p}=$ $\mathrm{f}(\mathrm{m})$ resp. $\Delta \mathrm{p}=\mathrm{f}(\mathrm{V})$ of the observed model, it should be to test at first suitable procedures of the solution. Because of a very high number of necessary simulations, the procedure should be the shortest, but with warranted and verified results. Main conclusions are mentioned in Par. 2.

The prepared method was used at first for the socalled basic model - Par. 3 and later for various shape variations of it - Par. 4.

From the many received results, the summary characteristics are evaluated and finally simple graphs, too, for a prediction of the new model series of valves. At the end, it should be noted that any simulating model is a better or worse approach to the reality, only, which should be tested by measuring on the real piece and so to make sure that the results of a simulation are in the conformity, either very good, or acceptable, or approximate only.

\section{Acknowledgment}

Our acknowledgment is given to VUTS Liberec - Centre for Development in Machinery Research for the support in the framework of the grant NPU-LO1213, "National program of sustainability", granted by the Czech ministry for education, youth and sport.

\section{References}

1. Vortex valve, patent US 3219048 from 23.11.1965

2. Jarman, D., Butler, D., Tabor, G., Andoh, R.: Modelling of vortex flow control at high drainage flow rates, ICE proceedings - Engineering and computational mechanics, Vol. 168 issue EM1

3. Andoh, R.Y.G., Faram, M.G., Osey, K.: Vortex flow controls in integrated stormwater management for urban environments, Proc. of the 6th int. water sensitive urban design conference and hydropolis, Perth Australia, 2009 